\title{
Universal Pion Freeze-out Phase-Space Density
}

\author{
D. Ferenc, B. Tomášik, U. Heinz, Inst. f. Theor. Physik, Universität Regensburg
}

G. Bertsch has indicated [1] a possibility to measure the pion freeze-out phase-space density and thereby test the local thermal equilibrium in a pion source. In case of thermal equilibrium at temperature $T$, identical pions of energy $E$ would follow the Bose-Einstein distribution

$$
f=\frac{1}{e^{\frac{E}{T}}-1} .
$$

An average of this function over different phase-space regions is the quantity to be measured. When the $p_{T^{-}}$ spectrum is parameterized by an exponential with $T_{\text {eff }}(y)$ being the inverse slope parameter, averaging over the spatial coordinates yields

$$
\langle f\rangle\left(p_{T}, y\right)=\frac{\frac{\sqrt{\pi}}{2} \frac{\sqrt{\lambda_{\text {dir }}\left(p_{T}, y\right)}}{E_{p} T_{\text {eff }}^{2}(y)} \exp \left(-\frac{p_{T}}{T_{\text {eff }}(y)}\right) \frac{d n^{-}}{d y}(y)}{R_{s}\left(p_{T}, y\right) \sqrt{R_{o}^{2}\left(p_{T}, y\right) R_{l}^{2}\left(p_{T}, y\right)-R_{o l}^{4}\left(p_{T}, y\right)}}
$$

This equation comprises information from essentially two different classes of experimental results: the single particle momentum spectra $\left(d n^{-} / d y, T_{e f f}\right)$, and the two pion Bose-Einstein correlations $\left(R_{s}, R_{o}, R_{l}, R_{o l}, \lambda_{d i r}\right)$. We have calculated $\langle f\rangle\left(p_{T}, y\right)$ for the $\mathrm{S}-\mathrm{S}, \mathrm{S}-\mathrm{Cu}, \mathrm{S}-\mathrm{Ag}, \mathrm{S}-\mathrm{Au}, \mathrm{S}-\mathrm{Pb}$ and $\mathrm{Pb}-\mathrm{Pb}$ data from the experiments NA35 [2], NA49 [3], $\mathrm{NA} 44$ [4], and for the $\pi$-p data from the NA22 experiment [5] at CERN-SPS. From the results for $\langle f\rangle$ as a function of $p_{T}$, presented in Fig. 1, one may conclude:

\section{1. universal phase-space density}

All the nuclear collision data from the SPS in Fig. 11 are indistinguishably similar, in spite of a factor of $\sim 10$ difference in multiplicity density.

\section{2. agreement with Bose-Einstein distribution}

Using simultaneously pion spectra and pion correlations NA49 has disentangled thermal motion from collective expansion [3]. Taking the NA49 result for the local freezeout temperature $T=120 \mathrm{MeV}$ as the only parameter in Eq. (11) one indeed finds good agreement with the data. This is consistent with the thermal nature of the pion source, and inconsistent with the presence of a hypothetic pion condensate.

\section{3. radial flow}

Looking in more detail, one finds that the data indicate a somewhat slower decrease with increasing $p_{T}$ than the Bose-Einstein curve. This is most likely due to radial collective expansion which adds extra transverse momentum to particles, i.e. the local $\langle f\rangle$ values appear in the measurement at a $p_{T}$ that is higher than the local $\left\langle p_{T}\right\rangle$ in the source reference frame. A detailed study is under way.

\section{4. rapidity dependence}

A certain departure from the universal scaling is seen for the data at rapidities close to the projectile rapidity, both at AGS and SPS; moreover, the two results are consistent.

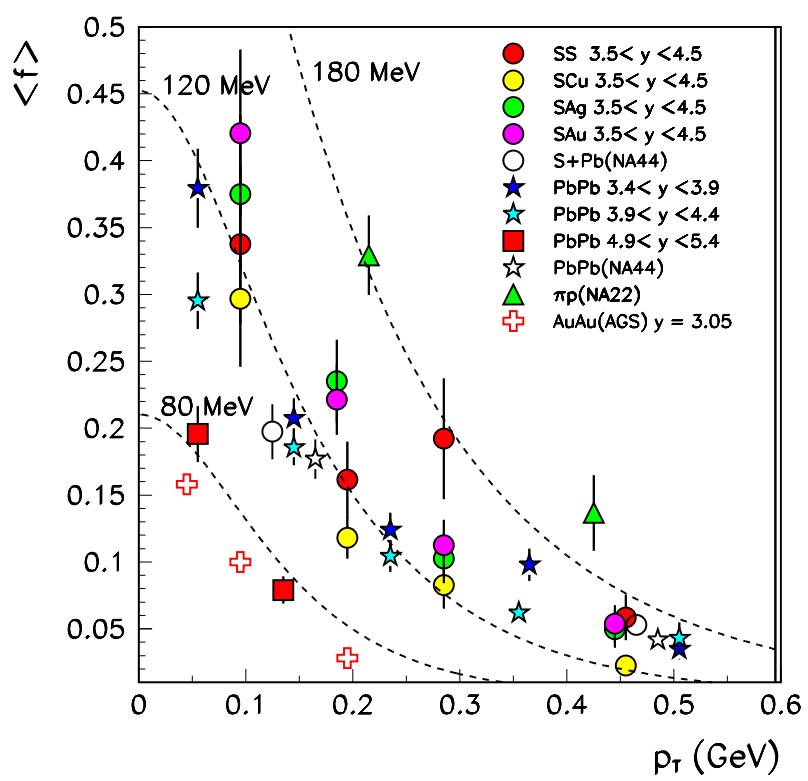

Figure 1: Phase-space density as a function of $p_{T}$ for different data sets. Heavy-ion data from SPS are indistinguishably similar, although they span over an order of magnitude range in multiplicity. A Bose-Einstein function (Eq. (11) is superimposed with the three choices of the local freeze-out temperature: $80 \mathrm{MeV}, 120 \mathrm{MeV}$ and 180 $\mathrm{MeV}$.

5. high temperature decoupling in $\pi-p$ collisions In contrast to freeze-out in nuclear collisions which takes place in two steps (chemical at $T \simeq 170-180 \mathrm{MeV}$ and thermal at $T \simeq 120 \mathrm{MeV}$ ), pion production in $\pi$-p collisions [5] is essentially immediate, without the second evolution stage, and therefore freeze-out temperatures of around $180 \mathrm{MeV}$ should be expected. The data [5] are indeed consistent with this expectation, as seen in Fig. 1.

\section{References}

[1] G.F. Bertsch, Phys. Rev. Lett. 72 (1994) 2349, and 77 (1996) 789e.

[2] NA35, T. Alber et al., Z. Phys. C66 (1995) 77, Phys. Rev. Lett. 74 (1995) 1303.

[3] NA49, H. Appelshäuser, Ph.D. Thesis, Univ. Frankfurt, 1997. H. Appelshäuser et al., Eur. Phys. J. C2 (1998) 661.

[4] NA44, H. Beker et al., Phys. Rev. Lett. 74 (1995) 3340. I.G. Bearden et al., Phys. Rev. C58 (1998) 1656.

[5] N.M. Agababyan et al., Z. Phys. C71 (1996) 405.

[6] E877, J. Barrette et al., Phys. Rev. Lett. 78 (1997) 2961. 